\title{
Research of Task Management and Resource Allocation in Cloud Computing
}

\author{
Prasad Devarasetty, Ch. Satyananda Reddy
}

\begin{abstract}
This paper presents the task management framework in which tasks are identified, analysed and grouped based on the user QoS requirements. As a next step, cost and time based scheduling algorithm is employed to select the suitable pair of resources and tasks. The performance of the proposed algorithm is tested with execution time and cost allocated to the resources. Two existing algorithms are considered along with proposed algorithm for performance comparison. The results proved the efficiency of the proposed algorithm.
\end{abstract}

Keywords - Cost, Time, Scheduling, cloud, agent etc.

\section{INTRODUCTION}

Cloud Computing has the ability to provide different types of services over geographical regions. Resource allocation in cloud is one of the complex procedures due to the phenomenon of searching best possible combinations of tasks and resources [1-5]. Various methods are developed to solve the problem of resource allocation because different types of on-demand services offered by cloud service providers' causes challenges to the customers for selecting the resources [6, 7-9]. The allocation of tasks to the resources is a complex task in cloud environment and it can be easily solved by Machine Learning concepts. These concepts allocate the resources based on the QoS parameters.

Resource allocation is represented as algorithms which improves the performance of the computing resources by minimizing the cost, energy consumption, execution time and improves the effectiveness [3]. However, allocating more tasks to single resource leads to performance degrades and it takes more time to execute the tasks which leads to user dissatisfaction. Resource scheduling mechanism allocates the tasks to suitable resources, and hence the applications can effectively utilize the resources which ultimately lead to scaling advantage [10].

In this paper, we concentrate on finding the best pair of task and resources according to the user requirements. In the cloud environment, the main QoS constraints are minimizing the execution time of tasks and reducing the cost of resources. This paper proposes cost and time based scheduling algorithm for achieving QoS requirements in cloud environment. The rest of the paper is organized as follows: Section 2 deals with literature survey and the contributions. Section 3 explains about the task management framework with problem statement. Section 4 deals with the cost and time based scheduling algorithm. A comparison is

Revised Manuscript Received on April 12, 2019.

Prasad Devarasetty,Department of Computer Science and Engineering DVR \&Dr HS MIC College of Technology, Vijayawada, Andhra Pradesh, India. (saiprasshad@ gmail.com)

Dr. Ch. Satyananda Reddy, Department of Computer Science and Systems Engineering, AU College of Engineering, Andhra University, Visakhapatnam, Andhra Pradesh, India. made with existing algorithms in section 5. Finally, section 6 concludes the research work.

\section{TASK MANAGEMENT FRAMEWORK}

In the cloud environment, resource allocation is the main module of resource management. It essentially allocates the resources to the tasks from the available pool of resources. Based on the user preference, the framework searches the suitable resources to the submitted workload. The resource allocation procedure is categorized in to four steps. In the initial step, the tasks are analysed and grouped based on the user requirements. As a next step, the suitable resources need to be identified from the pool of resources. In the third step, resources are allocated to the tasks based on the requirements of the user. In the final step, resources scheduling is performed based on the selection of appropriate scheduling policy by the user to achieve the optimal QoS [1-2]. Figure 1 shows the task management Framework and Figure 2 shows the flow diagram of task resource scheduling procedure.

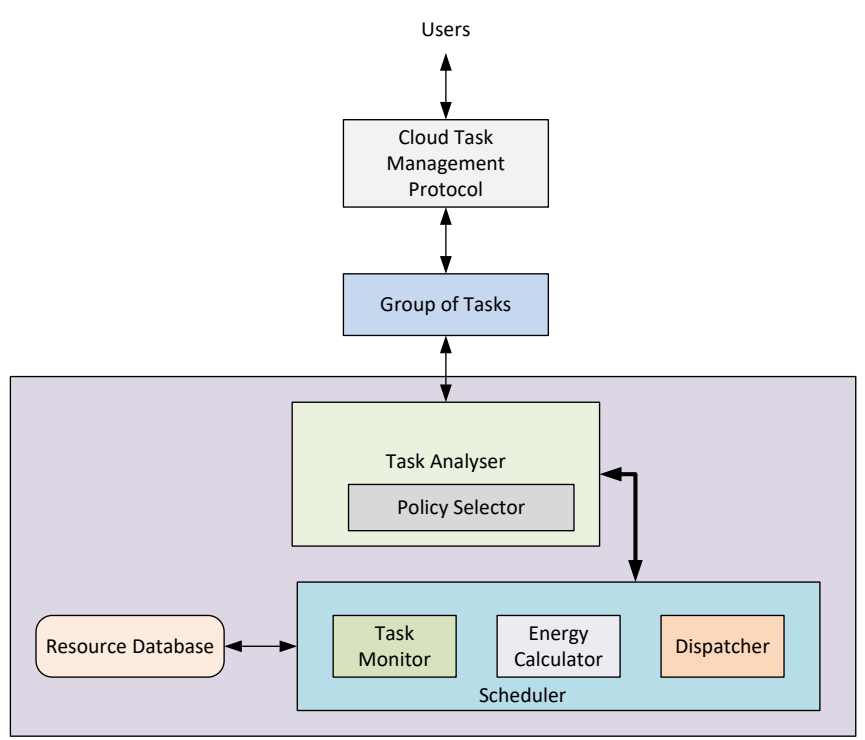

Figure 1: Task Management Framework [1]

The task management frameworks execute the task requests as follows [1]:

- The cloud users submit their tasks to the task management framework. As a next step, the user authentication and authorization is processed.

- The task management system requests the user to submit the requirements to execute the tasks.

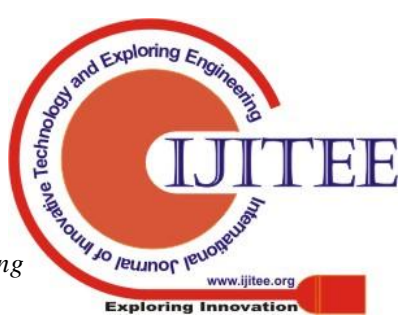


- The cloud verifies the task workload and searches for the available resources to meet the user requirements.

- The resources scheduling policy is employed by using the decision tree based on the submitted user requirements.

- At the time of task execution, the proposed framework checks the current task workload.

- If the required resource value is greater than the available resources, then it will identify the scheduling policy based on the cost and time appropriately.

- After executing the tasks successfully, the proposed framework releases the resources and allocates to the new tasks.

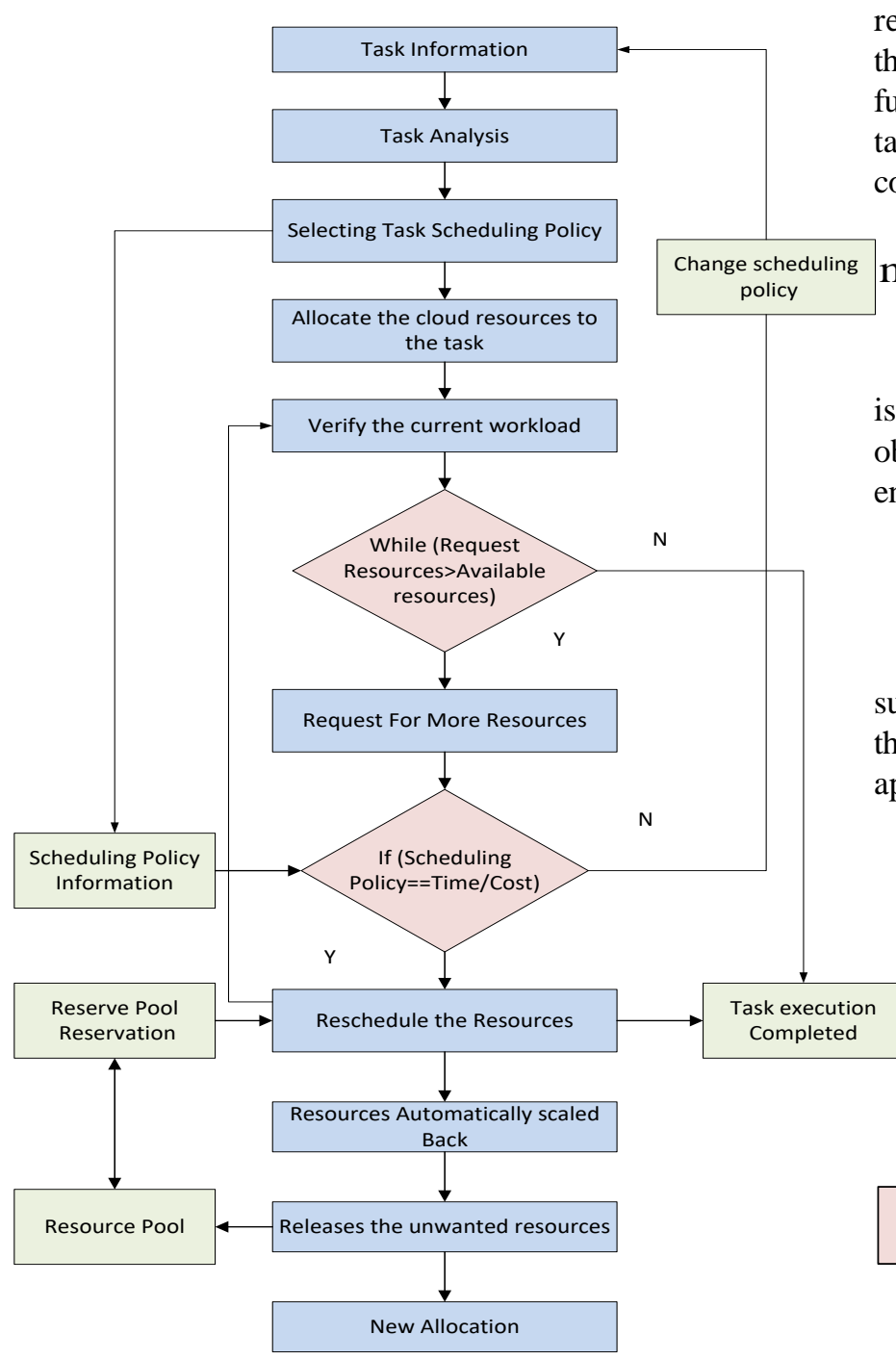

Figure 2: Flow diagram of Task Management Framework [1]

Cloud resource allocation is a complex procedure due to the identifying of best pair of task and resource based on the QoS requirements. The aim of the task analyser is to divide the tasks and the aim of the resource scheduler is to map the tasks to the resources effectively. In general, the cloud resources have the property of heterogeneity and they are in dynamic nature. The resources can leave or join the network at any time. The resource scheduling problem is expressed as follows: The set of independent tasks $\left\{\mathrm{t}_{1}, \mathrm{t}_{2} \ldots \mathrm{t}_{\mathrm{m}}\right\}$ to assign the resources $\left\{r_{1}, r_{2} \ldots r_{n}\right\}$ is considered. $T=\left\{t_{i} \mid\right.$
$1 \leq \mathrm{i} \leq \mathrm{m}\}$ is the collection of tasks and $\mathrm{m}$ is the total number of tasks, $\mathrm{R}=\left\{\mathrm{r}_{\mathrm{k}} \mid 1 \leq \mathrm{k} \leq \mathrm{n}\right\}$ represents the group of resources and $n$ denotes the number of resources. For the proposed framework, the following constraints need to be considered.

1. The tasks submitted by the user to execute the application should contain the unique task id.

2. The submitted task should be independent.

3. The arrival of tasks to the cloud is random and the tasks are placed in the scheduling queue for execution.

4. The objective function determines the execution time of the task on a resource.

\subsection{Objective function}

In cloud environment, the service provider needs to reduce the execution time and the cloud user needs to reduce the cost to execute the tasks. The main aim of the objective function is to minimize the cost and time for the submitted tasks. Eq. 1 shows the objective function for reducing the cost and time.

$\min f(x)=\sum_{m=1}^{n}\left(E S T_{x}\right)_{m} \times\left(C_{H}\right)_{m}$

Where $\mathrm{m}$ is the current task executing in the cloud, $E S T_{x}$ is the estimated time and $C_{H}$ is the cost per hour. The objective of the cloud service provider is to minimize the energy consumption and maximize the resource utilization.

\section{SCHEDULING ALGORITHM BASED ON THE DECISION-TREE APPROACH}

Decision tree presents the rule in the different structures such as hierarchal and sequential structure. Figure 3 shows the scheduling algorithm selection based on the decision tree approach.

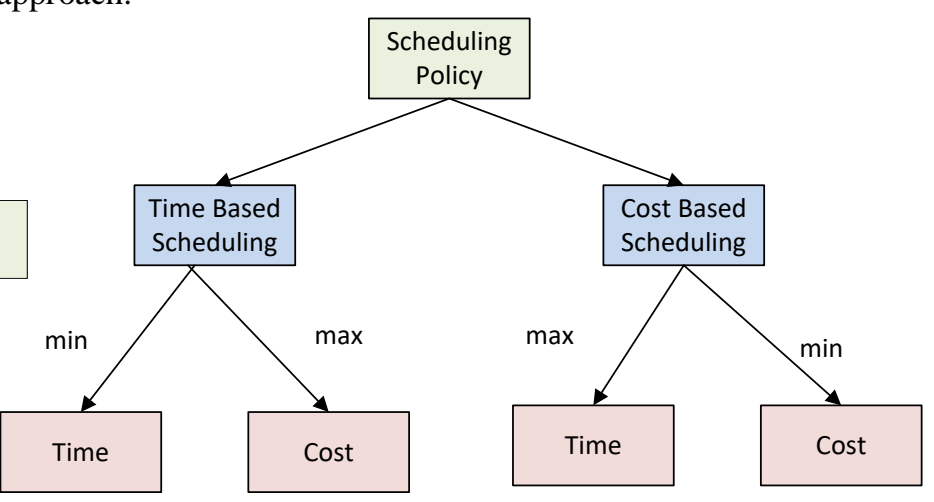

Figure 3: Scheduling Policy Selection based on the Decision tree Approach

\subsection{Cost and Time Based Scheduling Algorithm (CTSA):}

In the CTSA [1], the cloud service provider reduces the execution time, cost along with the energy consumption of the tasks. This algorithm uses Eqs. (2), (3) and (4) to calculate the total completion time (TCT), total cost (TC) and the time difference $\left(\mathrm{TM}_{\mathrm{d}}\right)$ to assign the resources to the tasks. The resource allocation agent identifies the missing deadlines of the tasks, recalculates the time difference and 
assigns the resources to the tasks for completing the task execution with in the assigned deadline. Algorithm 1 shows the cost and time based scheduling algorithm. The fitness function for the algorithm is given as follows.

$$
\begin{aligned}
& T C T=E T+C T \\
& T C=C C+C_{\text {min }} \\
& T M_{d}=D D-C u r_{t}
\end{aligned}
$$

Where ET represents the execution time, CT represents the communication time, $\mathrm{CC}$ represents the communication cost, $\mathrm{C}_{\min }$ represents the minimum cost, DD represents the defined deadline for the task and Cur $_{\mathrm{i}}$ represents the current time taken for executing the task

The complexity of the algorithm is given in eq. 4 which depends on the requested resources (w) and rescheduling process of the tasks.

$$
\beta=\left(S T+E T+T_{\text {sus }}+T_{\operatorname{Re} s}\right)
$$

Here ST represents the starting time of the tasks, ET represents the ending time of the tasks, $\mathrm{T}_{\text {sus }}$ represents the suspending time of the tasks and $\mathrm{T}_{\text {Res }}$ represents the resume time of the tasks.

\section{SIMULATION ENVIRONMENT\& RESULTS}

The proposed algorithm is simulated with CloudSim tool kit. 2000 independent tasks are generated randomly. For each task, the estimated cost, deadline and scheduling algorithm is taken as attributes. Table 1 shows the parameters of resources and tasks that have been considered for simulation.

Table 1: Parameters for resources and tasks

\begin{tabular}{|c|c|}
\hline Parameters & Value \\
\hline Number of Tasks & 2000 \\
\hline Number of Resources & $50-200$ \\
\hline Size of task & $10-30 \mathrm{MB}$ \\
\hline Bandwidth & $1000-2000 \mathrm{~B} / \mathrm{S}$ \\
\hline Cost per execution of task & $\$ 2-\$ 5$ \\
\hline PE Ratings & $1000-\mathrm{MIPS}$ \\
\hline Memory Size & $2 \mathrm{MB}-20 \mathrm{MB}$ \\
\hline Number of PEs/ machine & 1 \\
\hline
\end{tabular}

Figure 4 shows the execution time of CTSA, CTC and DBD-CTO algorithms. The execution time of three scheduling algorithms is tested with different tasks using same resources. The DBD-CTO recorded 1,123 s to execute the 2000 tasks whereas the CTC and CTSA recorded 1097s and $987 \mathrm{~s}$ to execute the tasks. It is observed that the execution time of CTSA is less compared to the CTC and DBD-CTO algorithms.

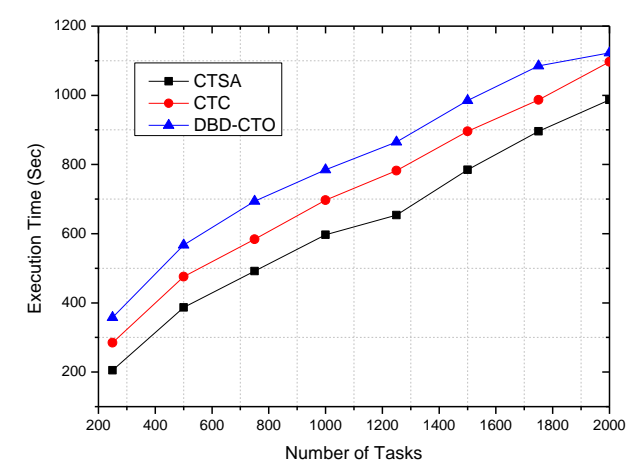

Figure 4: Comparison of Execution time with respect to Number of Tasks

Figure 5 shows the cost of three algorithms at different tasks. The DBD-CTO algorithm spent $129 \$$ to execute the tasks, CTC spent $114 \$$ and CTSA spent $105 \$$. From the figure, it is observed that CTSA has less expenditure compared to all algorithms.

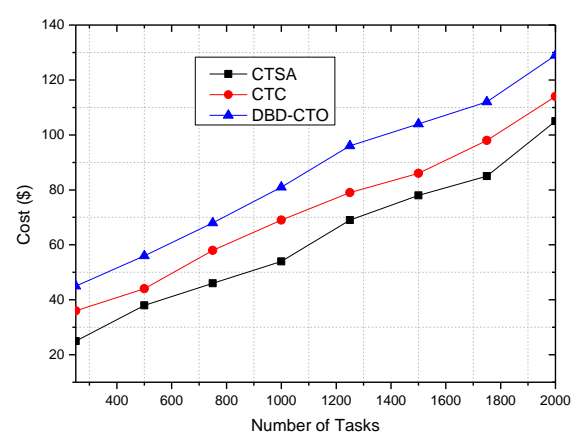

Figure 5: Comparison of cost with respect to number of tasks

Figure 6 shows the comparison of algorithms with respect to increase in terms of resources by keeping the tasks submitted to cloud constant. In this simulation, 2000 tasks are submitted with different number of resources. The results proved that the execution time decreases by increasing the number of resources. CTSA algorithm performs better and had better execution time compared to the CTC and DBD-CTO.

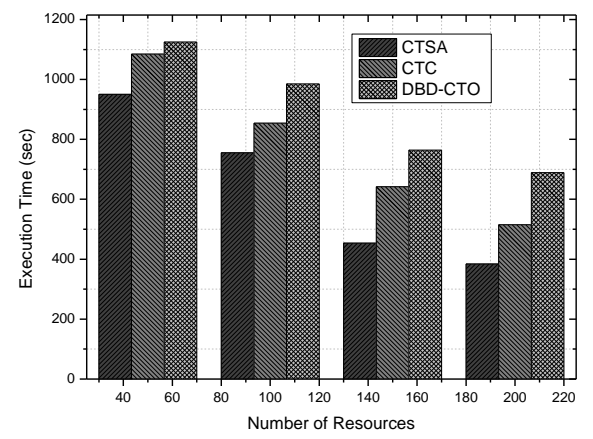

Figure 6: Comparison of Execution time with Number of Resources 
Figure 7 represents the comparison of cost with number of resources. The cost of resources minimizes while increase in the number of resources. From the figure; it is observed that CTSA executed the tasks with minimum cost compared with the CTC and DBD-CTO. The cost for renting 50 resources is around $50 \$$ and the cost for 100 resources will cost around $159 \$$ on CTSA algorithm. Therefore, it saves around $30 \%$ of the cost compared to the fixed resources.

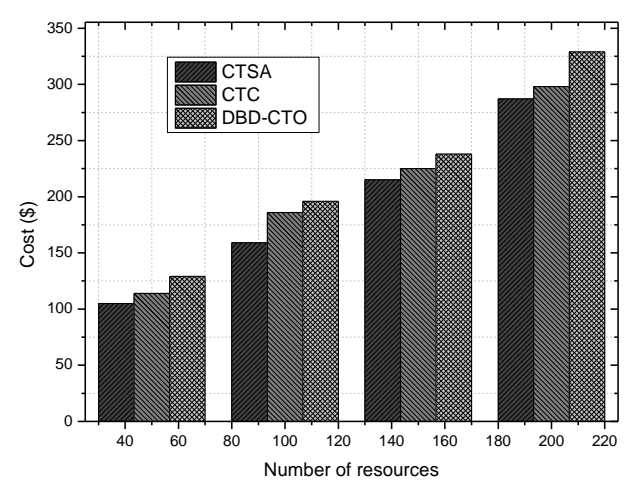

Figure 7: Comparison of Cost with Number of Resources

\section{CONCLUSION}

This research discussed the important characteristics of Cloud Computing along with the challenges faced by resource scheduling algorithms. This paper also presented the task management framework which can identify different task and analysed their QoS requirements. The cost and time based resource scheduling algorithm is proposed for managing the scheduling policy to identify the better task and resource pairs. The simulation results showed that the proposed algorithm had better performance in terms of execution time and cost compared to the existing algorithms such as CTC and DBD-CTO.

\section{REFERENCES}

1. Singh, S., \&Chana, I. (2015). QRSF: QoS-aware resource scheduling framework in cloud computing. The Journal of Supercomputing, 71(1), 241-292.

2. Singh, Sukhpal, and InderveerChana. "A survey on resource scheduling in cloud computing: Issues and challenges." Journal of grid computing 14, no. 2 (2016): 217 264.

3. Devarasetty, P., \& Reddy, S. (2019). Genetic algorithm for quality of service based resource allocation in cloud computing. Evolutionary Intelligence, 1-7.

4. Liu K, Jin H, Chen J, Liu X, Yuan D, Yang Y (2010) A compromised-time-cost scheduling algorithm in swindew-c for instance-intensive cost-constrained workflows on a Cloud computing platform. Int $\mathbf{J}$ High Perform ComputAppl 24(4):445-456.

5. J. Huang, Y. Liu, and Q. Duan, "Service provisioning in virtualization based cloud computing: Modeling and optimization," in Proc. of IEEE Global Communications Conference (GLOBECOM), Dec 2012, pp.1710-1715.

6. S. Chaisiri, B. Lee, and D. Niyato, "Optimization of Resource Provisioning Cost in Cloud Computing," IEEE Transactions on Services Computing, vol. 5, no. 2, pp. 164-177, June 2012.

7. LD, DhineshBabu, and P. Venkata Krishna. "Honey bee behavior inspired load balancing of tasks in cloud computing environments." Applied Soft Computing 13.5 (2013): 22922303.
8. Misra, S., Krishna, P. V., Kalaiselvan, K., Saritha, V., \&Obaidat, M. S. (2014). Learning automata-based QoS framework for cloud IaaS. IEEE Transactions on Network and Service Management, 11(1), 15-24.

9. Babu, L. D., \& Krishna, P. V. (2013). Versatile time-cost algorithm (VTCA) for scheduling non-preemptive tasks of time critical workflows in cloud computing systems. International Journal of Communication Networks and Distributed Systems, 11(4), 390-411.

10. Krishna, P. V., Misra, S., Saritha, V., Raju, D. N., \&Obaidat, M. S. (2017, May). An efficient learning automata based task offloading in mobile cloud computing environments. In 2017 IEEE International Conference on Communications (ICC) (pp. 1-6). IEEE. 